\title{
EVOLUÇÃO DIFERENCIAL HÍBRIDA COM PROGRAMAÇÃO QUADRÁTICA APLICADA AO PROBLEMA DE DESPACHO ECONÔMICO DE ENERGIA ELÉTRICA
}

\author{
Leandro dos Santos Coelho* \\ leandro.coelho@pucpr.br
}

\author{
Viviana Cocco Mariani ${ }^{\dagger}$ \\ viviana.marianiepucpr.br
}

*Laboratório de Automação e Sistemas,

Programa de Pós-Graduação em Engenharia de Produção e Sistemas, LAS / PPGEPS, Grupo Produtrônica Centro de Ciências Exatas e de Tecnologia (CCET), Pontifícia Universidade Católica do Paraná (PUCPR)

Rua Imaculada Conceição, 1155, CEP 80215-030, Curitiba, PR, Brasil.

${ }^{\dagger}$ Programa de Pós-Graduação em Engenharia Mecânica, PPGEM

Centro de Ciências Exatas e de Tecnologia (CCET), Pontifícia Universidade Católica do Paraná (PUCPR)

Rua Imaculada Conceição, 1155, CEP 80215-030, Curitiba, PR, Brasil.

\section{RESUMO}

Neste artigo propõe-se uma nova metodologia híbrida para resolução do problema de despacho econômico de energia elétrica com o efeito do ponto de válvula. O método híbrido proposto integra evolução diferencial (ED) com a técnica de programação quadrática seqüencial (SQP). A ED é utilizada como otimizador global e a SQP é utilizada para o ajuste fino da otimização, realizada pela ED. A metodologia híbrida e suas variantes são validadas para sistemas teste de 3,13 e 40 unidades geradoras térmicas com funções incrementais de custo de combustível que levam em consideração o efeito de carregamento devido ao ponto de válvula. O método híbrido proposto supera e providencia soluções promissoras, em termos de eficiência, se comparadas com as obtidas pela ED e SQP sozinhas bem como de outras técnicas propostas na literatura para o problema de despacho econômico de carga com o efeito do ponto de válvula.

PALAVRAS-CHAVE: evolução diferencial, programação

Artigo submetido em 20/12/2004

1a. Revisão em 26/08/2006

2a. Revisão em 19/10/2006

Aceito sob recomendação do Editor Associado

Prof. Ivan Nunes Da Silva quadrática seqüencial, despacho econômico, efeito do ponto de válvula, método híbrido de otimização.

\section{ABSTRACT}

This paper proposes a new hybrid methodology for solving the economic load dispatch problem with valve-point effect. The proposed hybrid method integrates the differential evolution (DE) with the sequential quadratic programming (SQP) technique. The DE is the global optimizer and the SQP is used to fine tune of DE run. The hybrid methodology and its variants are validated for test systems consisting of 3,13 and 40 thermal units with incremental fuel cost function takes into account the valve-point loadings effects. The proposed hybrid method outperforms and provides quality solutions in terms of efficiency compared with those obtained from DE and SQP alone and other existing techniques for load dispatch problem with valve-point effect.

KEYWORDS: differential evolution, sequential quadratic programming, economic load dispatch, valve-point effect, hybrid optimization method. 


\section{INTRODUÇÃO}

Os sistemas elétricos são interconectados de forma a obter os benefícios de custos mínimos de geração, confiança máxima e melhores condições operacionais, tais como compartilhamento de reserva de energia, aprimoramento da estabilidade e operação sobre situações de emergência. Neste contexto, o problema de otimização do despacho econômico de energia elétrica é relevante para atendimento de requisitos de qualidade e eficiência na geração de energia elétrica.

O objetivo básico do problema de despacho econômico da geração de energia elétrica é o escalonamento das saídas das unidades de geração conveniadas para encontrar a demanda de carga consumidora a um custo mínimo de operação, satisfazendo a todas unidades e as restrições de igualdade e desigualdade impostas pelo problema (Abido, 2003). Quando o problema de despacho econômico trata de um intervalo de tempo simples, ele é referido como um problema de despacho econômico estático. Já o problema de despacho econômico dinâmico considera um número finito de intervalos de despacho acoplados com a previsão de carga para providenciar uma trajetória de geração "ótima" seguindo uma demanda variável de carga (Chowdhury \& Rahman, 1990).

Muitos dos problemas de otimização em sistemas de potência, incluindo os de despacho econômico, possuem características complexas e não-lineares com a presença, muitas vezes, de restrições de igualdade e desigualdade. Desde que o problema de despacho econômico foi introduzido, diversos métodos têm sido utilizados para resolver este problema.

Abordagens convencionais para resolução de problemas de despacho econômico incluem método iterativo $\lambda$ (Wood \& Wollenberg, 1984; Chen \& Wang, 1993), técnicas baseadas em gradiente (Wood \& Wollenberg, 1984; Lee et al., 1984), estrutura hierárquica por partes de funções quadráticas (Lin \& Viviani, 1984), método dos pontos interiores (Granville, 1992), programação linear (El-Keib \& Ding, 1994; Farag et al., 1995) e programação dinâmica (Shoults \& Venkatesh, 1986; Yang \& Chen, 1989; Liang \& Glover, 1992). Entretanto, muitas das abordagens convencionais usadas em problemas de despacho econômico podem não ser aptas para providenciar uma solução ótima e, muitas vezes, a solução fica retida a armadilhas de mínimos locais.

A literatura tem apresentado alguns estudos referentes a utilização de metodologias da inteligência artificial clássica para problemas de despacho econômico, tais como busca tabu (Lin et al., 2002), simulated annealing (Wong \& Fung, 1993) e sistemas especialistas (Wang \& Shahidepour, 1992). Neste contexto, as aplicações de técnicas de inteligência artificial usando principalmente a sub-área de inteligência computacional, a citar as aplicações de redes neurais (Park et al., 1993; Lee et al., 1998; Yalcinoz \& Short, 1998; Lee \& Kim, 2002), nuvem de partículas (Park et al., 2005; Coelho \& Mariani, 2006), algoritmos evolutivos ou evolucionários (Miranda $e t$ al., 1998), sistemas nebulosos (Song et al., 1997; Attaviriyanupap et al., 2004) e sistemas híbridos inteligentes (Chang \& Fu, 1998) têm sido abordadas com sucesso devido a suas habilidades de procurar soluções próximas a solução ótima global.

No contexto específico de algoritmos evolutivos (AEs), diversos estudos recentes têm sido apresentados visando a concepção de algoritmos de otimização estocásticos eficientes baseados em algoritmos genéticos (Walters \& Sheble, 1993; Alander, 1995; Chen \& Chang, 1995; Sheble \& Brittig, 1995; Xu et al., 1996; Su \& Chiang, 2004), programação evolucionária (Yang et al., 1996; Wong \& Yurkevich, 1998; Park et al., 1999; Jayabarathi et al., 2000; Kumarappan \& Mohan, 2003; Sinha et al., 2003; Venkatesh et al., 2003) e algoritmos evolutivos híbridos (Wong \& Wong, 1994; Li et al., 1997; Das \& Patvardham, 1999; Ruangpayoongsak et al., 2002).

A contribuição deste artigo é descrever e avaliar uma nova metodologia híbrida para resolução do problema de despacho econômico de carga considerando o efeito do ponto de válvula. O método híbrido proposto integra uma abordagem recente e eficiente de algoritmo evolutivo denominado evolução diferencial (ED) para a etapa de busca global (fase de evolução) combinada com uma técnica de programação quadrática seqüencial (Sequential Quadratic Programming, SQP) para a etapa de busca local (fase de aprendizado).

A concepção híbrida de otimização adotada, neste artigo, é também denominada na literatura de AE híbrido, AE com busca local, algoritmo memético ou otimização baseada em evolução Lamarckiana (Moscato \& Norman, 1992; Whitley et al., 1994; Ku et al., 2000; Wellock \& Ross, 2001). A vantagem da utilização de um método híbrido de otimização de ED e SQP está na melhoria da velocidade de convergência do algoritmo de otimização como um todo em relação a busca na vizinhança de um ponto promissor. A solução final obtida pelo método híbrido tende a ser mais próxima da solução ótima, em média, que a obtida pela ED ou pela SQP quando atuam isoladamente.

A metodologia híbrida e suas variantes são validadas para três estudos de caso conhecidos da literatura para 3, 13 e 40 unidades geradoras térmicas (Walter \& Sheble, 1993; Wong \& Wong, 1994; Sinha et al., 2003) considerando-se o efeito de válvula. Os resultados obtidos são analisados e comparados com outros apresentados na literatura, que ressaltam a eficiência da abordagem híbrida proposta neste artigo.

O artigo é organizado da seguinte forma. A formulação do problema de despacho econômico de energia elétrica é detalhada na seção 2. Na seção 3 são apresentados os fundamentos da ED e SPQ nas formas de concepção isolada e híbrida. 
Além disso, comentários sobre o tratamento de restrições são apresentados. A descrição dos três problemas de despacho econômico testados e uma análise dos resultados de otimização obtidos são apresentados na seção 4. Finalizando o artigo, a conclusão e as perspectivas de futuros trabalhos são apresentadas na seção 5, respectivamente.

\section{FORMULAÇÃO DO PROBLEMA DE DESPACHO ECONÔMICO}

O tipo de problema de despacho econômico, abordado neste artigo, pode ser descrito matematicamente com uma função objetivo e duas restrições. As restrições representadas pelas equações (1) e (2) devem ser satisfeitas,

$$
\begin{gathered}
\sum_{i=1}^{n} P_{i}-P_{L}-P_{D}=0 \\
P_{i}^{\min } \leq P_{i} \leq P_{i}^{\max }
\end{gathered}
$$

A equação (1) representa as restrições de igualdade do balanço de potência (isto é, balanço entre suprimento e demanda), enquanto a expressão (2) representa as restrições de desigualdade relativas aos limites da capacidade de geração de potência de cada unidade geradora, onde $P_{i}$ é a saída para a unidade geradora $i$ (em $\mathrm{MW}) ; n$ é o número de geradores presente no sistema; $P_{D}$ é a demanda de carga total (em MW); $P_{L}$ são as perdas de transmissão (em MW) e $P_{i}^{\min }$ e $P_{i}^{\max }$ são respectivamente as saídas de operação mínimas e máximas da unidade geradora $i(\mathrm{em} \mathrm{MW})$. O custo total de combustível deve ser minimizado conforme representado na equação (3),

$$
\min f=\sum_{i=1}^{n} F_{i}\left(P_{i}\right)
$$

onde $F_{i}$ é a função custo de combustível para a unidade geradora $i(\mathrm{em} \$ / \mathrm{h})$, que é definida pela equação,

$$
F_{i}\left(P_{i}\right)=a_{i} P_{i}^{2}+b_{i} P_{i}+c_{i}
$$

onde $a_{i}, b_{i}$ e $c_{i}$ são coeficientes de custo do gerador $i$ (Park et al., 2005).

A figura 1 mostra a curva de desempenho para uma unidade térmica típica com 4 válvulas de turbinas a calor. A linha cheia representa a curva aproximada por funções quadráticas, representadas pela equação (4). Entretanto, esta curva não representa as características de entrada e saída reais de geradores térmicos. Para modelar a função custo dos geradores em uma forma mais prática, a linha tracejada da figura 1 deve ser utilizada. As variações na curva de entrada e saída (linha tracejada) expressam o resultado da grande variação nas perdas devido aos efeitos de trefilação que ocorrem em cada válvula de admissão de calor que começa a abrir. Este fenômeno é denominado de ponto de efeito de válvula (IEEE Committee Report, 1971). A função custo, neste caso, é obtida baseada na introdução de "ripples" nas curvas de taxa de calor para modelagem mais precisa do efeito de ponto de válvula, pois as unidades de geração com múltiplas turbinas de calor exibem grande variação nas curvas de taxa de calor nas funções custo do combustível. A curva contém nãolinearidade de alta ordem e descontinuidade devido ao efeito do ponto de válvula, conforme mostrado na figura 1, e pode ser refinada por uma função seno. Então, a equação (4) para o cálculo do custo total pode ser modificada para considerar o efeito do ponto de válvula (Wood \& Wollenberg, 1984), tal que

$$
\tilde{F}_{i}\left(P_{i}\right)=F\left(P_{i}\right)+\left|e_{i} \operatorname{sen}\left(f_{i}\left(P_{i}^{\min }-P_{i}\right)\right)\right|
$$

ou

$$
\tilde{F}_{i}\left(P_{i}\right)=a_{i} P_{i}^{2}+b_{i} P_{i}+c_{i}+\left|e_{i} \operatorname{sen}\left(f_{i}\left(P_{i}^{\min }-P_{i}\right)\right)\right|
$$

onde $e_{i}$ e $f_{i}$ são constantes do efeito do ponto de válvula dos geradores. Conseqüentemente, o custo total de combustível que deve ser minimizado, conforme representado na equação (3), é modificado para

$$
\min f=\sum_{i=1}^{n} \tilde{F}_{i}\left(P_{i}\right)
$$

onde $\tilde{F}_{i}$ é a função custo para a unidade geradora $i(\mathrm{em} \$ / \mathrm{h})$, que é definida pela equação (6). Nos exemplos abordados, neste artigo, são desconsideradas as perdas de transmissão $P_{L}$, portanto, neste caso $P_{L}=0$.

\section{OTIMIZAÇÃO APLICADA À RESOLU- ÇÃO DO PROBLEMA DE DESPACHO ECONÔMICO}

Os métodos de otimização têm duas formas de configuração: os métodos determinísticos e os métodos estocásticos. As técnicas determinísticas tendem a buscar um ponto de mínimo (quando o problema é de minimização) no espaço de busca baseadas na informação dada pelo gradiente da função objetivo (função custo). A eficiência destas técnicas depende 


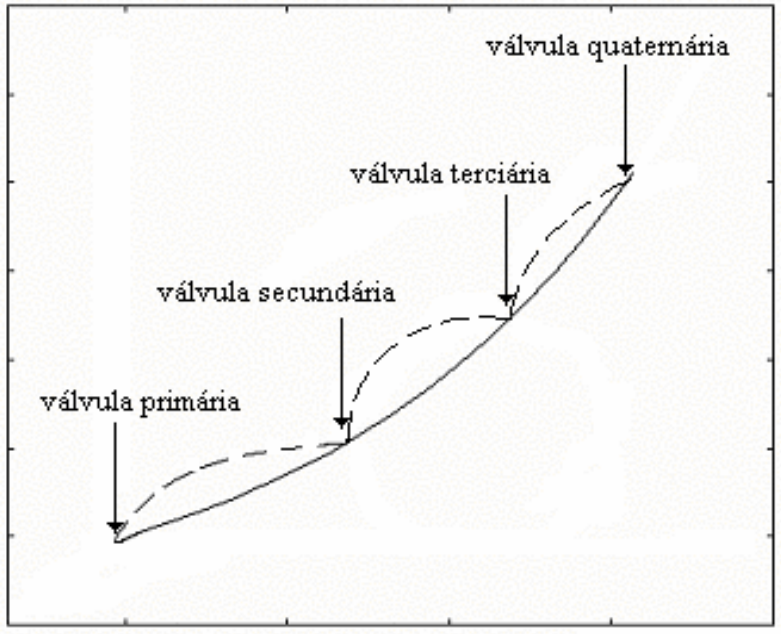

geração (MW)

Figura 1: Curva de entrada e saída de geradores térmicos para um exemplo de 4 válvulas.

de diversos fatores, tais como: a solução inicial, a precisão da avaliação da direção descendente, o método utilizado para executar a busca em linha e o critério de parada adotado. A solução obtida é geralmente um ponto de mínimo local, que pode ser mínimo global se a função apresentar apenas uma moda. As duas desvantagens principais dos métodos determinísticos, dos quais a programação quadrática faz parte, são a necessidade de avaliações do gradiente e falta da garantia do mínimo global para problemas multimodais.

Os métodos estocásticos, dos quais os AEs fazem parte, não necessitam do cálculo do gradiente e são aptos a encontrar a solução global. Contudo, o número de avaliações da função objetivo, necessárias para encontrar a solução, é geralmente maior que o número requerido pelos métodos determinísticos (Vasconcelos et al., 1999).

Os AEs são ferramentas promissoras para busca, otimização, aprendizado de máquina e para resolução de problemas de projeto. Estes algoritmos utilizam a evolução simulada buscando soluções para problemas complexos (Bäck et al., 1997; Whitley, 2001). Os AEs são baseados em uma população de indivíduos, onde cada um representa um ponto de busca no espaço de soluções potenciais de um dado problema. Os AEs possuem alguns procedimentos de seleção baseados na aptidão (fitness) dos indivíduos, operadores de cruzamento (crossover) e mutação.

Os AEs incluem algoritmos genéticos, programação evolucionária, estratégias evolutivas, programação genética, entre outras variantes (Bäck et al., 1997). Dentre as variantes de AEs destaca-se o recentemente proposto algoritmo de ED. A
ED foi desenvolvida por Price \& Storn (1995) e visa a busca por melhores resultados com uma abordagem um pouco diferente da utilizada nos algoritmos genéticos, mas com similaridades de implementação com as estratégias evolutivas. $\mathrm{Na}$ ED a mutação não é realizada por uma função densidade de probabilidade (Storn, 1999). Algumas das potencialidades da ED são: (i) a rapidez de convergência da otimização e (ii) a facilidade de implementação.

A seguir são detalhados os fundamentos e potencialidades da ED, SQP e o método híbrido combinando ED e SQP.

\subsection{Evolução diferencial}

Neste artigo enfoca-se a ED, proposta originalmente por Storn \& Price (1995), que apesar de apresentar conceitos simples é de fácil implementação, robusta e eficiente para a minimização de funções não-lineares e não-diferenciáveis no espaço contínuo. Na ED, os parâmetros da função a ser otimizada são codificados com variáveis reais (representação em ponto flutuante) na população e são realizadas mutações simples com uma operação aritmética simples. Storn (1997) relatou resultados impressionantes que mostram que a ED supera outros AEs (simulated annealing adaptativo, Nelder e Mead com annealing, algoritmo genético breeder, estratégia evolutiva e equações diferenciáveis estocásticas) para abordagens de minimização em relação ao número de avaliações necessárias localizando o mínimo global de diversas funções teste consolidadas na literatura.

A escolha da ED para resolução de problemas de despacho econômico foi baseada nas seguintes características (Cheng \& Hwang, 2001):

- é um algoritmo de busca estocástica que apresenta menor tendência de se concentrar em mínimos locais, pois a busca pelo ótimo global é feita através da manipulação de uma população de soluções ou, em outras palavras, por uma busca simultânea em diferentes áreas do espaço de soluções;

- é eficiente para problemas de otimização de funções objetivo que não requerem informações relativas à derivadas;

- permite que os parâmetros de entrada e saída sejam representados como ponto flutuante sem nenhum esforço computacional adicional (como é o caso da representação binária dos algoritmos genéticos);

- não necessita manter um tamanho grande de população.

As diferentes variantes da ED reportadas na literatura (Storn, 1997) são classificadas usando a seguinte notação: 
$\mathrm{ED} / \alpha / \beta / \delta$, onde $\alpha$ indica o método de seleção dos indivíduos genitores que formarão o vetor base que passará pela operação de mutação, o $\beta$ indica o número de vetores de diferenças utilizados para perturbar os indivíduos do vetor base e $\delta$ indica o mecanismo de cruzamento utilizado para geração da população de indivíduos descendentes. A sigla bin indica que a operação de cruzamento é controlada por uma série de experimentos binomiais.

A implementação de ED adotada, neste artigo, é a $\mathrm{ED} / \mathrm{rand} / 1 /$ bin. Neste caso, cada variável de um indivíduo (vetor) da população é representada por um valor real (ponto flutuante) e o seu procedimento de otimização para o problema de despacho econômico é regido pelas seguintes etapas:

(i) gerar a população inicial: gerar de forma aleatória com distribuição uniforme uma população inicial (geração $G=0$ ) de soluções factíveis, onde é garantido por regras de "reparo" que os valores atribuídos as variáveis estão dentro das fronteiras delimitadas pelo projetista;

$$
\begin{aligned}
P_{i}^{j}(G=0)=\lim _{\text {inf }}\left(P_{i}\right) & +\operatorname{rand}_{i}[0,1] * \\
& *\left\{\lim _{\text {sup }}\left(P_{i}\right)-\lim _{\text {inf }}\left(P_{i}\right)\right\}
\end{aligned}
$$

onde $j=1, \ldots, M$ indivíduos, $M$ é o tamanho da população $(M>4), n$ é a dimensão da solução, $\lim _{i n f}\left(P_{i}\right)$ e $\lim _{\text {sup }}\left(P_{i}\right)$ são os limites inferior e superior de valores admissíveis para a variável $x_{i}$, respectivamente, e $\operatorname{rand}_{i}[0,1]$ gera um número aleatório, com distribuição uniforme, no intervalo entre 0 e 1 .

(ii) avaliar a população: para cada indivíduo é avaliada a função de aptidão (observação: os detalhes do cálculo da função de aptidão com penalização $q$ são apresentados na seção 3.4 deste artigo);

(ii) selecionar indivíduos: um indivíduo $P_{i}^{j}(G)$, da $i$-ésima unidade geradora na $G$-ésima geração, é selecionado de forma aleatória, para ser substituído e outros três diferentes indivíduos $r_{1}, r_{2}, r_{3}$ (valores inteiros do indivíduo $i$ da população) são selecionados aleatoriamente como genitores;

(iii) operação de mutação: um destes três indivíduos é selecionado como genitor principal (por exemplo, $r_{1}$ ) e com alguma probabilidade, cada variável do genitor principal é modificada. Neste caso, pelo menos uma variável deve ser alterada. A modificação é realizada adicionando ao valor atual da variável um fator de mutação, $F$, regida pela diferença entre dois valores desta variável nos outros dois genitores, ou seja,

$$
P_{i}(G+1)=P_{i, r_{1}}(G)+F \cdot\left[P_{i, r_{2}}(G)-P_{i, r_{3}}(G)\right]
$$

Em outras palavras, o vetor denominado genitor principal é modificado baseado no vetor de variáveis de dois outros genitores.

(iv) operação de cruzamento: seguindo a operação de mutação, o cruzamento é aplicado na população. Para cada vetor que sofreu mutação, $P_{i}(G+1)$, um índice $r n b r(i) \in\{1,2, \cdots, n\}$ é aleatoriamente escolhido, e um vetor tentativa, $\mathrm{Pt}_{i}(G+1)$, é gerado tal que

$$
P t_{i, y}(G+1)=\left\{\begin{array}{l}
P_{i, q}(G+1), \\
\text { se } \operatorname{randb}(\mathrm{y}) \leq C R \text { ou } \mathrm{y}=\operatorname{rnbr}(i) \\
P_{i, q}(G), \\
\text { se } \operatorname{randb}(\mathrm{y})>C R \text { ou } \mathrm{y} \neq \operatorname{rnbr}(i)
\end{array}\right.
$$

onde $i=1, . ., n$ é um índice de indivíduo da população e $y=1, . ., n$ é a posição do indivíduo $n-$ dimensional. Para decidir se o vetor $P t_{i}(G+1)$ deve ser um membro da população na próxima geração, este é comparado com o vetor $P_{i}(G+1)$ correspondente. Se o vetor $P t_{i}(G+1)$ apresenta uma função de aptidão melhor que a escolhida à substituição, ele a substitui; caso contrário, o vetor $P_{i}(G+1)$ é mantido na população.

(iv) critério de parada: incrementar uma unidade ao valor de $G$; após ir para o passo (ii) até que um critério de parada seja atendido. Neste artigo o critério de parada é o número máximo de gerações, $G_{\max }$.

Na ED, $F>0$ é um parâmetro real, que controla a amplificação da diferença entre dois indivíduos e para evitar a estagnação é usualmente projetado no intervalo $[0,1 ; 2]$; $\operatorname{randb}(y)$ é a $y$-ésima avaliação de um número aleatório com distribuição uniforme no intervalo [0,1] e $C R$ é a taxa de cruzamento (crossover rate) no intervalo [0, 1]. O desempenho da ED depende de três variáveis: o tamanho de população $M$, o fator de mutação $F$ e a taxa de cruzamento $C R$.

As configurações de $\mathrm{ED} / \mathrm{rand} / 1 /$ bin testadas, neste trabalho, foram:

- $\operatorname{ED}(1): M=30, F=0,4, C R=0,8$ e penalização $q=$ 200 (detalhes sobre o tratamento de restrições e forma de penalização são descritos na seção 3.4);

- $\mathrm{ED}(2): M=30, F=0,4, C R=0,8$ e penalização $q=500$;

- $\operatorname{ED(3):~} M=30, F=0,4, C R=0,8$ e penalização $q=$ 1000 ; 
- $\mathrm{ED}(4): M=30, F=0,4, C R=0,8$ e penalização $q=$ 2000

- $\mathrm{ED}(5): M=30, F$ entre 0,4 e 1,0 (escolhido aleatoriamente a cada geração com distribuição uniforme), $C R=$ 0,8 e penalização $q=200$.

- $\mathrm{ED}(6): M=30, F$ entre 0,4 e 1,0 (escolhido aleatoriamente a cada geração com distribuição uniforme), $C R=$ 0,8 e penalização $q=200$. Neste caso, é usado um algoritmo cultural normativo (Reynolds, 1994; Jin \& Reynolds, 1999) a cada $\phi$ gerações. O conhecimento normativo contém o intervalo para as variáveis de decisão onde "boas" soluções têm sido encontradas, de forma a mover novas soluções para aquele intervalo. Os limites inferior, $N_{\text {inf }}(G)$, e superior, $N_{\text {sup }}(G)$, das soluções $P_{i}^{j}(G)$ para implementação de conhecimento normativo são dados pelas expressões:

$$
\left[N_{\text {inf }}(G), N_{\text {sup }}(G)\right]=\left[\min P_{i}(G), \max P_{i}(G)\right]
$$

e a equação (9) da ED é modificada para:

$$
\begin{aligned}
& \operatorname{Caso} P_{i, r_{1}}(G)<N_{\mathrm{inf}}(G) \text { : } \\
& P_{i}(G+1)=P_{i, r_{1}}(G)+F \cdot\left[P_{i, r_{2}}(G)-P_{i, r_{3}}(G)\right]
\end{aligned}
$$

Caso $P_{i, r_{1}}(G)>N_{\text {sup }}(G)$ :

$$
P_{i}(G+1)=P_{i, r_{1}}(G)-F \cdot\left[P_{i, r_{2}}(G)-P_{i, r_{3}}(G)\right]
$$

Nos outros casos

$$
P_{i}(G+1)=P_{i, r_{1}}(G)+F \cdot\left[P_{i, r_{2}}(G)-P_{i, r_{3}}(G)\right]
$$

\subsection{Programação quadrática seqüencial}

Uma eficiente e precisa solução para o problema de despacho econômico não depende apenas do tamanho do problema, em termos do número de restrições e das variáveis de projeto, mas também depende das características da função objetivo e das restrições. Quando ambas as funções objetivo e restrições são funções lineares das variáveis de projeto, o problema de despacho econômico é conhecido como um problema de programação linear. O problema de programação quadrática $(\mathrm{QP})$ refere-se a minimização ou maximização de uma função objetivo quadrática que é linearmente restrita.

O problema mais difícil de resolver é o problema de programação não-linear em que a função objetivo e as restrições podem ser funções não-lineares das variáveis de projeto. A solução deste último problema requer um procedimento iterativo para obter uma direção de busca em cada iteração. Esta direção pode ser encontrada pela solução de um subproblema QP. Os métodos para resolver estes problemas são comumente referidos como SQP desde que um subproblema QP é resolvido em cada iteração maior, também são conhecidos como programação quadrática iterativa, programação quadrática recursiva ou método de variável métrica restrita.

Os problemas resolvidos, no presente trabalho, são de programação quadrática e não-linear porque a função objetivo é quadrática e não-linear, respectivamente, conforme as equações (4) e (6), contudo as restrições de igualdade e desigualdade são lineares, e serão resolvidos através do procedimento iterativo SQP. O SQP é, em muitos casos, superior aos demais métodos de programação não-linear para otimização com restrições, possuindo vantagens em termos de eficiência, precisão e sucesso na obtenção de soluções, em um grande número de problemas teste disponíveis na literatura (Fletcher, 1987; Boggs \& Tolle, 2000; Rodríguez-Toral et al., 2001; Arsham et al., 2003).

A implementação do SQP consiste de três estágios principais, que são brevemente descritos a seguir:

(i) calcular uma aproximação da matriz Hessiana da função Lagrangeana usando um método quase-Newton;

(ii) gerar o sub-problema de QP;

(iii) proceder em uma direção de descida usando um método de busca em linha.

Formulando o subproblema de QP para o problema enunciado nas equações (1), (2) e (7) tem-se,

$$
\min f(P)=\frac{1}{2} \mathrm{~d}_{\mathrm{k}}^{\mathrm{T}} \mathrm{H}_{\mathrm{k}} \mathrm{d}_{\mathrm{k}}+\vec{\nabla} \mathrm{f}\left(\mathrm{P}_{\mathrm{k}}\right)^{\mathrm{T}} \mathrm{d}_{\mathrm{k}}
$$

sujeito a

$$
\begin{aligned}
& c\left(P_{k}\right)+\vec{\nabla} c\left(P_{k}\right)^{T} d_{k}=0 \\
& P_{\text {min }} \leq P_{k}+d_{k} \leq P_{\max }
\end{aligned}
$$

onde $d_{k} \in \operatorname{IR}^{n}$ e $P_{k} \in \operatorname{IR}^{n} ; H_{k}$ é a matriz Hessiana da função Lagrangeana na $k$-ésima iteração,

$$
L(P, \lambda)=f(P)+c(P)^{T} \lambda
$$

onde $d_{k}$ é a direção de busca na $k$-ésima iteração; $P_{k}$ é o vetor de potência real na $k$-ésima iteração; e $c\left(P_{k}\right)$ é a restrição de igualdade apresentada na formulação de despacho econômico. 
Em cada iteração uma aproximação da matriz Hessiana, positiva definida, da função Lagrangeana, $H$, é calculada numericamente. Dos métodos que usam a informação do gradiente, os mais vantajosos são os métodos quase-Newton. Esses métodos dependem da informação da curvatura em cada iteração para formular um problema quadrático da forma da equação (15).

Os métodos quase-Newton evitam o cálculo direto da matriz Hessiana numericamente, o que envolve um grande número de cálculos, e usam o comportamento da função $f(P)$ e de seu gradiente para desenvolver informações para a curvatura e construir uma aproximação para $H$, através de uma técnica apropriada.

Alguns métodos para atualização da matriz Hessiana têm sido desenvolvidos. Geralmente, o método de Broyden, Fletcher, Goldfarb e Shanno (BFGS) é um dos métodos mais eficazes e, portanto, é utilizado neste trabalho. Assim, a atualização da matriz Hessiana através do BFGS é regida pela equação

$$
\begin{gathered}
H_{k+1}=H_{k}+\frac{q_{k} q_{k}^{T}}{q_{k}^{T} s_{k}}-\frac{H_{k}^{T} s_{k}^{T} s_{k} H_{k}}{s_{k}^{T} H_{k} s_{k}} \\
s_{k}=P_{k+1}-P_{k}, \\
q_{k}=\vec{\nabla} L\left(P_{k+1}, \lambda_{k+1}\right)-\vec{\nabla} L\left(P_{k}, \lambda_{k+1}\right)
\end{gathered}
$$

onde $\lambda$ é uma estimativa dos multiplicadores de Lagrange. Em cada iteração do subproblema de QP a direção $d_{k}$ é calculada usando a equação (15). A solução obtida é usada para formar a nova iteração expressa por,

$$
P_{k+1}=P_{k}+\alpha_{k} d_{k}
$$

onde $\alpha$ é o comprimento do passo determinado para produzir uma redução considerável na função mérito Lagrangeana aumentada

$$
L_{A}(P, \lambda, \rho)=f(P)-\lambda^{T} c(P)+\frac{\rho}{2} c(P) c(P)
$$

onde $\rho$ é um escalar não-negativo.

\subsection{Evolução diferencial híbrida com SQP}

A configuração de abordagens compostas por técnicas determinísticas, estas hibridizadas com técnicas estocásticas, é uma alternativa promissora em otimização e que deve ser avaliada. A ED e a SQP possuem potencialidades complementares. A ED é robusta e pode ser projetada para buscas em um amplo espaço de busca (busca global). A SQP é freqüentemente apropriada para convergir rapidamente para uma região em torno de um mínimo local para problemas de despacho econômico de energia elétrica considerando o efeito do ponto de válvula. A SQP pode ser incorporada a ED visando a obtenção de um aprimoramento da otimização quanto a busca local.

Neste contexto, para obter os benefícios da configuração de otimização híbrida, uma forma eficiente é executar, inicialmente, a ED para localizar a região de "ótimo" global e a SQP para a busca local. A SQP avalia alguns dos melhores indivíduos da ED (ou mesmo somente o melhor indivíduo) como soluções iniciais do problema. No caso, a SQP obtém melhores soluções que os melhores indivíduos testados. Estes indivíduos, na próxima geração da ED, são substituídos pelas soluções obtidas pela SQP.

Neste artigo, foram testadas seis formas de hibridização:

- ED-SQP(1): a cada geração $G$ é verificado se houve melhora do valor de $f *$ do melhor indivíduo da população da ED. Em caso afirmativo, é realizada uma busca local usando SQP com estimativa inicial igual ao melhor indivíduo da população da ED. Caso a SQP ao final da sua otimização obtenha uma estimativa de $f<f^{*}$, esta estimativa de solução substituirá o melhor indivíduo da população da ED na próxima geração $G+1$.

- ED-SQP(2): idem a ED-SQP(1). Entretanto, se a cada $\beta$ gerações não houve melhoria do melhor indivíduo da população da ED, é gerada uma nova solução factível (aleatoriamente com distribuição uniforme). Esta nova solução factível é utilizada como estimativa inicial para a realização de uma busca local usando SQP. Caso a SQP ao final da sua otimização obtenha uma estimativa de $f<f *$, esta estimativa de solução substituirá o melhor indivíduo da população da ED na próxima geração $G+1$.

- ED-SQP(3): idem a ED-SQP(2). Entretanto, é aplicado um algoritmo cultural normativo (Reynolds, 1994; Jin $\&$ Reynolds, 1999) a cada $\phi$ gerações.

- ED-SQP(4): após o critério de parada de otimização da ED ter sido atingido é realizada uma busca local usando SQP com estimativa inicial igual ao melhor indivíduo da população da ED;

- ED-SQP(5): após o critério de parada de otimização da ED (usando conhecimento normativo a cada $\phi$ gerações) ter sido atingido é realizada uma busca local usando SQP com estimativa inicial igual ao melhor indivíduo da população da ED;

- ED-SQP(6): idem a ED-SQP(5). Entretanto, é realizada uma análise de diversidade dos valores de $f$ de toda a 
população da ED a cada geração. Os membros da população com valor de $f$ menor que $1 \%$ do melhor valor de $f$ na geração são reiniciados (gera-se uma nova solução factível aleatoriamente e com distribuição uniforme).

\subsection{Tratamento de restrições}

A metodologia de tratamento de restrições é dividida em duas etapas. A primeira etapa visa à obtenção de soluções para as variáveis de decisão dentro dos limites inferior $\left(\lim _{\text {inf }}\right)$ e superior $\left(\lim _{\text {sup }}\right)$ impostos pelo projetista, isto é, $x \in\left[\lim _{\text {inf }}, \lim _{\text {sup }}\right]$ da equação (2). Neste caso, quando uma restrição de limite inferior não é satisfeita aplica-se a regra de "reparo",

$$
\begin{aligned}
P_{i}^{j}(G+1)= & P_{i}^{j}(G)+w \cdot \operatorname{rand}_{i}[0,1] \cdot \\
& \cdot\left\{\lim _{\sup }\left(P_{i}^{j}(G)\right)-\lim _{\inf }\left(P_{i}^{j}(G)\right)\right\}
\end{aligned}
$$

De forma análoga, no caso de que uma restrição de limite superior não seja satisfeita utiliza-se a seguinte regra de reparo,

$$
\begin{aligned}
P_{i}^{j}(G+1)= & P_{i}^{j}(G)-w \cdot \operatorname{rand}_{i}[0,1] \cdot \\
& \cdot\left\{\lim _{\sup }\left(P_{i}^{j}(G)\right)-\lim _{\inf }\left(P_{i}^{j}(G)\right)\right\}
\end{aligned}
$$

onde $w \in[0,1]$ é um parâmetro de projeto (adota-se $w=$ $0,05)$ e rand $[0,1]$ é um valor aleatório gerado com distribuição uniforme entre 0 e 1 .

Na segunda etapa as variáveis de decisão são consideradas na igualdade da equação (1). Caso a igualdade não seja atendida, modifica-se a equação (7) para

$$
\min f=\sum_{i=1}^{n} \tilde{F}_{i}\left(P_{i}\right)+q \cdot\left|\sum_{i=1}^{n} P_{i}-P_{L}-P_{D}\right|
$$

onde $q$ é uma constante de penalidade aplicada à restrição de igualdade não atendida da equação (1) e $|\cdot|$ consiste do valor absoluto da expressão.

A abordagem de penalização usando a equação (24) é utilizada em todos os métodos de otimização apresentados neste trabalho, ou seja, ED, SQP e ED-SQP.

\section{EXEMPLOS DE APLICAÇÃO}

Para mostrar o desempenho relativo da ED, SQP e ED-SQP, três estudos de caso foram simulados. As simulações são validadas para sistemas teste de 3,13 e 40 unidades geradoras térmicas com funções incrementais de custo de combustível que levam em consideração o efeito de ponto de válvula. Os algoritmos para resolução dos exemplos foram implementados em ambiente computacional Matlab $6.5^{\circledR}$, da MathWorks, usando processador AMD Athlon de $1,1 \mathrm{GHz}$ com 128 MB de memória RAM.

Foram realizadas 50 simulações com cada abordagem de otimização testada. No caso da ED, a cada simulação os membros da população foram iniciados usando uma semente diferente de números aleatórios. Além disso, na ED foi utilizado um critério de parada $G_{\max }$ com valor 200,400 e 3000 para os casos 1, 2, e 3, respectivamente. O valor de $\phi=5$ foi adotado para todas as simulações e $\beta=20,5$ e 200 , para os casos 1,2 , e 3 , respectivamente. Em relação à $\mathrm{SQP}$, a cada simulação a estimativa inicial foi gerada usando uma semente de números aleatórios (geração com distribuição uniforme) diferente.

As soluções obtidas foram comparadas em tabelas que mostram as características de convergência e tempo computacional com cada abordagem. No final desta seção, os resultados obtidos são também comparados com outros métodos apresentados na literatura.

\subsection{Caso 1 com 3 unidades geradoras}

O estudo de caso 1 compreende três unidades geradoras. A demanda de potência esperada para ser determinada pelas três unidades geradoras é $P_{D}=850 \mathrm{MW}$. Os dados do sistema são apresentados na tabela 1 e também podem ser encontrados em Waters \& Sheble (1993).

Tabela 1: Dados para o caso 1, onde Ge é o número do gerador e as potências $P_{i}^{\min }$ e $P_{i}^{\max }$ são em MW.

\begin{tabular}{|c|c|c|c|c|c|c|c|}
\hline$G e$ & $P_{i}^{\min }$ & $P_{i}^{\max }$ & $a$ & $b$ & $c$ & $e$ & $f$ \\
\hline 1 & 100 & 600 & 0,001562 & 7,92 & 561 & 300 & 0,0315 \\
\hline 2 & 50 & 200 & 0,004820 & 7,97 & 78 & 150 & 0,063 \\
\hline 3 & 100 & 400 & 0,001940 & 7,85 & 310 & 200 & 0,042 \\
\hline
\end{tabular}

Os resultados obtidos para o caso 1 são apresentados nas tabelas 2 e 3. A SQP e as ED-SQP(1)-(6) obtiveram os melhores custos. Entretanto, a SQP foi a técnica com convergência mais rápida das estudadas. A $\mathrm{ED}-\mathrm{SQP}(6)$ foi a que obteve menor custo médio e desvio padrão nas simulações realizadas, no entanto, a ED-SQP(6) comparada com as EDSQP(1)-(5) foi a que apresentou maior tempo médio para re- 
Tabela 2: Resultados de convergência para o caso teste 1 ( 3 unidades geradoras com ponto de válvula) e $P_{D}=850 \mathrm{MW}$.

\begin{tabular}{|c|c|c|c|c|c|}
\hline técnica & $\begin{array}{c}\text { tempo } \\
\text { médio }(\mathrm{s})\end{array}$ & $\begin{array}{c}\text { custo } \\
\text { mínimo }(\$ / \mathrm{h})\end{array}$ & $\begin{array}{c}\text { custo médio } \\
(\$ / \mathrm{h})\end{array}$ & $\begin{array}{c}\text { desvio padrão } \\
\text { do custo }(\$ / \mathrm{h})\end{array}$ & $\begin{array}{c}\text { custo } \\
\text { máximo }(\$ / \mathrm{h})\end{array}$ \\
\hline $\mathrm{SQP} *$ & $\mathbf{0 , 2 2}$ & $\mathbf{8 2 3 4 , 0 7 4 0}$ & 8324,8968 & 109,4337 & 8571,7518 \\
\hline $\mathrm{ED}(1)$ & 1,10 & 8234,0741 & 8252,0978 & 35,9134 & 8386,5050 \\
\hline $\mathrm{ED}(2)$ & 1,05 & 8234,0741 & 8247,4603 & 24,9140 & 8343,9362 \\
\hline $\mathrm{ED}(3)$ & 1,09 & 8234,0741 & 8246,3324 & 25,1057 & 8343,9362 \\
\hline $\mathrm{ED}(4)$ & 1,04 & 8234,0746 & 8257,2138 & 38,3899 & 8343,9362 \\
\hline $\mathrm{ED}(5)$ & 1,80 & 8234,0749 & 8241,2114 & 15,1879 & 8343,9370 \\
\hline $\mathrm{ED}(6)$ & 2,14 & 8234,0749 & 8241,1491 & 4,6246 & 8263,4828 \\
\hline $\mathrm{ED}-\mathrm{SQP}(1)$ & 2,99 & $\mathbf{8 2 3 4 , 0 7 4 0}$ & 8240,1109 & 15,3910 & 8343,9361 \\
\hline $\mathrm{ED}-\mathrm{SQP}(2)$ & 4,13 & $\mathbf{8 2 3 4 , 0 7 4 0}$ & 8234,3592 & 1,4103 & $\mathbf{8 2 4 1 , 2 2 2 3}$ \\
\hline $\mathrm{ED}-\mathrm{SQP}(3)$ & 5,07 & $\mathbf{8 2 3 4 , 0 7 4 0}$ & 8234,2517 & 1,0666 & 8241,5875 \\
\hline ED-SQP(4) & 1,80 & $\mathbf{8 2 3 4 , 0 7 4 0}$ & 8238,4672 & 4,1277 & 8250,2051 \\
\hline ED-SQP(5) & 2,48 & $\mathbf{8 2 3 4 , 0 7 4 0}$ & 8239,4724 & 4,4437 & 8250,4864 \\
\hline ED-SQP(6) & 5,14 & $\mathbf{8 2 3 4 , 0 7 4 0}$ & $\mathbf{8 2 3 4 , 2 2 0 3}$ & $\mathbf{1 , 0 0 4 7}$ & 8241,7957 \\
\hline
\end{tabular}

Tabela 3: Melhor solução para o estudo de caso 1 obtido usando ED-SQP(1-6) e SQP.

\begin{tabular}{|c|c|}
\hline potência & geração \\
\hline$P_{1}$ & 300,26418 \\
\hline$P_{2}$ & 400,00000 \\
\hline$P_{3}$ & 149,73583 \\
\hline$\sum_{i=1}^{3} P_{i}$ & 850,00000 \\
\hline
\end{tabular}

alizar a otimização. A ED, quando utilizada de forma isolada, obteve resultados próximos, mas inferiores aos obtidos pela SQP e as ED-SQP(1)-(6), provando ser uma abordagem promissora para busca global mas apresenta dificuldades na busca local.

\subsection{Caso 2 com 13 unidades geradoras}

Este problema consiste de treze unidades geradoras. Em relação ao caso 1 , a complexidade e a não-linearidade do problema são aumentadas. Neste caso a demanda de potência a ser encontrada pelas treze unidades geradoras é $P_{D}=1800 \mathrm{MW}$. Os dados do sistema são apresentados na tabela 4 e também podem ser encontrados em Wong \& Wong (1994) e Sinha et al. (2003).

Os resultados obtidos para o caso 1 são apresentados nas ta-
Tabela 4: Dados para o caso 2, onde Ge é o número do gerador e as potências $P_{i}^{\min }$ e $P_{i}^{\max }$ são em MW.

\begin{tabular}{|c|c|c|c|c|c|c|c|}
\hline$G e$ & $P_{i}^{\min }$ & $P_{i}^{\max }$ & $a$ & $b$ & $c$ & $e$ & $f$ \\
\hline 1 & 0 & 680 & 0,00028 & 8,10 & 550 & 300 & 0,035 \\
\hline 2 & 0 & 360 & 0,00056 & 8,10 & 309 & 200 & 0,042 \\
\hline 3 & 0 & 360 & 0,00056 & 8,10 & 307 & 150 & 0,042 \\
\hline 4 & 60 & 180 & 0,00324 & 7,74 & 240 & 150 & 0,063 \\
\hline 5 & 60 & 180 & 0,00324 & 7,74 & 240 & 150 & 0,063 \\
\hline 6 & 60 & 180 & 0,00324 & 7,74 & 240 & 150 & 0,063 \\
\hline 7 & 60 & 180 & 0,00324 & 7,74 & 240 & 150 & 0,063 \\
\hline 8 & 60 & 180 & 0,00324 & 7,74 & 240 & 150 & 0,063 \\
\hline 9 & 60 & 180 & 0,00324 & 7,74 & 240 & 150 & 0,063 \\
\hline 10 & 40 & 120 & 0,00284 & 8,60 & 126 & 100 & 0,084 \\
\hline 11 & 40 & 120 & 0,00284 & 8,60 & 126 & 100 & 0,084 \\
\hline 12 & 55 & 120 & 0,00284 & 8,60 & 126 & 100 & 0,084 \\
\hline 13 & 55 & 120 & 0,00284 & 8,60 & 126 & 100 & 0,084 \\
\hline
\end{tabular}

belas 5 e 6.

Nota-se que a SQP foi a abordagem de otimização testada com tempo médio menor para convergir. Os resultados com a SQP foram excelentes, o que pode ser notado pela repetibilidade obtida e pelo pequeno desvio padrão obtido nas 50 simulações. Da mesma forma que para o caso 1, a SQP e as ED-SQP(1)-(6) obtiveram os melhores custos. O menor custo médio foi obtido pela ED-SQP(6). Entretanto, o menor 
Tabela 5: Resultados de convergência para o caso teste 2 (13 unidades geradoras com ponto de válvula) e $P_{D}=1800 \mathrm{MW}$.

\begin{tabular}{|c|c|c|c|c|c|}
\hline técnica & $\begin{array}{c}\text { tempo } \\
\text { médio }(\mathrm{s})\end{array}$ & $\begin{array}{c}\text { custo } \\
\text { mínimo }(\$ / \mathrm{h})\end{array}$ & $\begin{array}{c}\text { custo médio } \\
(\$ / \mathrm{h})\end{array}$ & $\begin{array}{c}\text { desvio padrão } \\
\text { do custo }(\$ / \mathrm{h})\end{array}$ & $\begin{array}{c}\text { custo } \\
\text { máximo }(\$ / \mathrm{h})\end{array}$ \\
\hline $\mathrm{SQP}$ & $\mathbf{0 , 3 5}$ & $\mathbf{1 7 9 6 4 , 0 7 5 8}$ & $\mathbf{1 7 9 6 4 , 0 7 5 8}$ & $7,5246 \cdot 10^{-5}$ & 17944,8108 \\
\hline $\mathrm{ED}(1)$ & 4,08 & 17968,9537 & 17991,5667 & 14,7097 & 18035,1268 \\
\hline $\mathrm{ED}(2)$ & 4,06 & 17975,9964 & 18010,3311 & 18,5485 & 18048,2421 \\
\hline $\mathrm{ED}(3)$ & 4,12 & 17977,6722 & 18017,2263 & 18,5133 & 18051,2012 \\
\hline $\mathrm{ED}(4)$ & 4,10 & 17973,7392 & 18018,1550 & 20,9311 & 18057,2775 \\
\hline $\mathrm{ED}(5)$ & 4,00 & 17997,8411 & 18048,6729 & 25,1611 & 18134,6454 \\
\hline $\mathrm{ED}(6)$ & 6,86 & 17980,2180 & 18048,1203 & 25,6583 & 18118,4213 \\
\hline $\mathrm{ED}-\mathrm{SQP}(1)^{\#}$ & 0,40 & $\mathbf{1 7 9 6 4 , 0 7 5 8}$ & $\mathbf{1 7 9 6 4 , 0 7 5 8}$ & $5,0857 \cdot 10^{-5}$ & 17964,8107 \\
\hline $\mathrm{ED}-\mathrm{SQP}(2){ }^{\#}$ & 1,57 & $\mathbf{1 7 9 6 4 , 0 7 5 8}$ & $\mathbf{1 7 9 6 4 , 0 7 5 8}$ & $\mathbf{1 , 5 5 6 5 \cdot 1 0 ^ { - 6 }}$ & $\mathbf{1 7 9 6 4 , 8 1 0 4}$ \\
\hline $\mathrm{ED}-\mathrm{SQP}(3)^{\#}$ & 0,42 & $\mathbf{1 7 9 6 4 , 0 7 5 8}$ & $\mathbf{1 7 9 6 4 , 0 7 5 8}$ & $1,1340 \cdot 10^{-4}$ & 17964,8112 \\
\hline $\mathrm{ED}-\mathrm{SQP}(4)^{\#}$ & 0,43 & $\mathbf{1 7 9 6 4 , 0 7 5 8}$ & $\mathbf{1 7 9 6 4 , 0 7 5 8}$ & $8,3797 \cdot 10^{-5}$ & 17964,8110 \\
\hline $\mathrm{ED}-\mathrm{SQP}(5)^{\#}$ & 0,44 & $\mathbf{1 7 9 6 4 , 0 7 5 8}$ & $\mathbf{1 7 9 6 4 , 0 7 5 8}$ & $6,6607 \cdot 10^{-5}$ & 17964,8110 \\
\hline $\mathrm{ED}^{\#} \mathrm{SQP}(6)^{\#}$ & 0,43 & $\mathbf{1 7 9 6 4 , 0 7 5 8}$ & $\mathbf{1 7 9 6 4 , 0 7 5 8}$ & $1,0541 \cdot 10^{-3}$ & 17964,8111 \\
\hline
\end{tabular}

\# foi usada na ED uma população de 10 indivíduos e $G_{\max }=10$.

Tabela 6: Melhor solução para o estudo de caso 2 obtido usando ED-SQP(1-6) e SQP.

\begin{tabular}{|c|c|c|c|c|c|c|c|}
\hline potência & Geração & potência & geração & potência & geração & potência & geração \\
\hline$P_{1}$ & 628,3121 & $P_{5}$ & 60,0000 & $P_{9}$ & 109,8576 & $P_{13}$ & 55,0000 \\
\hline$P_{2}$ & 222,7723 & $P_{6}$ & 109,8690 & $P_{10}$ & 40,0000 & $\sum_{i=1}^{13} P_{i}$ & 1800,0000 \\
\hline$P_{3}$ & 149,5955 & $P_{7}$ & 109,8528 & $P_{11}$ & 40,0000 & & \\
\hline$P_{4}$ & 109,8647 & $P_{8}$ & 109,8748 & $P_{12}$ & 55,0000 & & \\
\hline
\end{tabular}

desvio padrão foi obtido pela ED-SQP(2). A ED apresentou resultados razoáveis, mas com alto custo computacional.

\subsection{Caso 3 com 40 unidades geradoras}

Este problema consiste de quarenta unidades geradoras. Em relação ao caso 2, a complexidade, não-linearidade e mínimos locais do problema são aumentados. Neste caso a demanda de potência a ser encontrada pelas quarenta unidades geradoras é $P_{D}=10500 \mathrm{MW}$. Os dados do sistema são apresentados na tabela 7 e também podem ser encontrados em Sinha et al. (2003).

Os resultados obtidos para o caso 1 são apresentados nas tabelas 8 e 9. A SQP, como nos casos 1 e 2 , foi a técnica mais rápida, no entanto não conseguiu obter a solução ótima e apresentou elevado desvio padrão nas 50 simulações realizadas. Nota-se, portanto, que a SQP para o caso 3 foi muito afetada pela estimativa inicial. A ED-SQP(4) foi a abordagem que obteve o menor custo para o problema de despacho econômico. Entretanto, foi a ED-SQP(5) que obteve melhor custo médio, menor desvio padrão e menor custo máximo entre as técnicas utilizadas. Neste contexto, a influência da concepção de conhecimento normativo (algoritmo cultural) na ED-SQP(5) foi decisivo para obtenção deste desempenho. A ED(6) obteve resultados competitivos com a SQP, no entanto com tempo computacional aproximadamente três vezes maior.

Na tabela 10 é apresentado um estudo comparativo de resultados obtidos neste artigo em relação a outros apresentados na literatura. Nota-se que para o caso 1 , os resultados da SQP e ED-SQP(1) a (6) foram iguais aos obtidos por abordagens de programação evolucionária, programação evolucionária híbrida com SQP, nuvem de partículas híbrida com SQP. No entanto, para os casos 2 e 3, os resultados obtidos neste trabalho de $f=17964,0758$ e $f=121715,4900$, respectivamente, foram melhores que de outros artigos apresentados na literatura. 
Tabela 7: Dados para o caso 3, onde Ge é o número do gerador e as potências $P_{i}^{\min }$ e $P_{i}^{\max }$ são em MW.

\begin{tabular}{|c|c|c|c|c|c|c|c|}
\hline$G e$ & $P_{i}^{\min }$ & $P_{i}^{\max }$ & $a$ & $b$ & $c$ & $e$ & $f$ \\
\hline 1 & 36 & 114 & 00690 & 6,73 & 94,705 & 100 & 0,084 \\
\hline 2 & 6 & 4 & & 6,73 & 05 & 100 & 0,084 \\
\hline 3 & 60 & 120 & 28 & 7,07 & 54 & 100 & 0,084 \\
\hline 4 & 80 & 190 & & 818 & 369,03 & 150 & 0,063 \\
\hline 5 & 47 & & & 5,35 & & 120 & 0,077 \\
\hline 6 & 68 & 140 & 142 & 8,05 & 222,33 & 100 & 0,084 \\
\hline 7 & 110 & 300 & 0,00357 & 8,03 & 278,71 & 200 & 0,042 \\
\hline 8 & 135 & 300 & 0,00492 & 6,99 & 391,98 & 200 & 0,042 \\
\hline 9 & 135 & 300 & 0,00573 & 6,60 & 455,76 & 200 & 0,042 \\
\hline 10 & 130 & 300 & 0,00605 & 12,90 & 722,82 & 200 & 0,042 \\
\hline 11 & 94 & 375 & 515 & 12,90 & 635,20 & 200 & 0,042 \\
\hline 12 & 94 & 375 & 0,00569 & 12,80 & 654,69 & 200 & 0,042 \\
\hline 13 & 125 & 500 & 421 & 12,50 & 913,40 & 300 & 0,035 \\
\hline 1 & 125 & 500 & 752 & 8,84 & 1760,4 & 300 & 0,035 \\
\hline 1 & 125 & 500 & 708 & 9,15 & 1728,3 & 300 & 0,035 \\
\hline 16 & 125 & 500 & 708 & 9,15 & 1728,3 & 300 & 0,035 \\
\hline 17 & 220 & 500 & 0313 & 7,97 & 647,85 & 300 & 0,035 \\
\hline 18 & 220 & 500 & 0,00313 & 7,95 & 649,69 & 300 & 0,035 \\
\hline 19 & 242 & 550 & 0313 & 7,97 & 647,83 & 300 &, 035 \\
\hline 20 & 242 & 550 & 313 & 7,97 & 647,81 & 300 & 0,035 \\
\hline 21 & 254 & 550 & 0,00298 & 6,63 & 785,96 & 300 & 0,035 \\
\hline 22 & 254 & 550 & 298 & 6,63 & 785,96 & 300 & 0,035 \\
\hline 23 & 254 & 550 & 284 & 6,66 & 794,53 & 300 &, 035 \\
\hline 24 & 254 & 550 & 284 & 6,66 & 794,53 & 300 & 0,035 \\
\hline 25 & 254 & 550 & 277 & 7,10 & 801,32 & 300 & 0,035 \\
\hline 26 & 254 & 550 & 77 & 7,10 & 32 & 300 & 0,035 \\
\hline 27 & 0 & 50 & 24 & 3,33 & 1055,1 & 120 & 0,077 \\
\hline 28 & 10 & 0 & 24 & 3,33 & 1055,1 & 120 & 0,077 \\
\hline 29 & 0 & 150 & 24 & 3,33 & 5,1 & 120 & 0,077 \\
\hline 3 & 7 & & & 5,35 & 9 & 120 & 0,077 \\
\hline 3 & 0 & 190 & & 6,43 & 92 & 150 & 0,063 \\
\hline 32 & 60 & 90 & 60 & 6,43 & 22 & 150 & 0,063 \\
\hline 33 & 60 & 190 & 0,00160 & 6,43 & 222,92 & 150 & 0,063 \\
\hline 34 & 90 & 200 & 0,00010 & 8,95 & 107,87 & 200 & 0,042 \\
\hline 35 & 90 & 200 & 0,00010 & 8,62 & 116,58 & 200 & 0,042 \\
\hline 36 & 90 & 200 & 0,00010 & 8,62 & 116,58 & 200 & 0,042 \\
\hline 37 & 25 & 110 & 0,01610 & 5,88 & 307,45 & 80 & 0,098 \\
\hline 38 & 25 & 110 & 0,01610 & 5,88 & 307,45 & 80 & 0,098 \\
\hline 39 & 25 & 110 & 0,01610 & 5,88 & 307,45 & 80 & 0,098 \\
\hline 40 & 242 & 550 & 0,00313 & 7,97 & 647,83 & 300 & 0,035 \\
\hline
\end{tabular}

\section{CONCLUSÃO E TRABALHOS FUTU- ROS}

Neste artigo, foi apresentada uma nova metodologia híbrida combinando ED e SQP para resolução do problema de despacho econômico de energia elétrica considerando o efeito do ponto de válvula. A ED foi utilizada para realizar a busca global, enquanto a SQP foi utilizada para sintonia fina das soluções obtidas pela ED.

Em relação ao procedimento de resolução do problema de despacho econômico de energia elétrica considerando o efeito do ponto de válvula, os resultados com a SQP e a ED-SQP para otimização das equações (1) e (2) foram iguais (caso 1) ou mesmo melhores (caso 2 e 3 ) que os apresentados em Walters \& Sheble (1993), Sinha et al. (2003), Victoire \& Jeyakumar (2004) e Part et al. (2005).

A SQP quando aplicada de forma isolada explora o espaço de busca rapidamente com a direção do gradiente e garante uma solução ótima local. O desempenho das seis abordagens de ED-SQP testadas nos três casos estudados foi animador, pois estas abordagens encontraram solução global de alta qualidade em tempo computacional aceitável. No entanto, em relação à SQP, a desvantagem foi o custo computacional maior da ED e da ED-SQP para obtenção da convergência.

A procura por um melhor compromisso entre exploitation (velocidade de convergência) e exploration (diversidade da população) é um tópico relevante das pesquisas com $\mathrm{ED}$, e será alvo de pesquisas futuras dos autores deste artigo, principalmente à concepção de abordagens híbridas com SQP para problemas de despacho econômico que incluem comportamento não-linear variante no tempo e a presença de múltiplos objetivos conflitantes e restrições de desigualdade.

\section{AGRADECIMENTOS}

Este trabalho contou com o apoio financeiro do Conselho Nacional de Desenvolvimento Científico e Tecnológico - CNPq (processo: 309646/2006-5/PQ). Agradecimentos também às valiosas e pertinentes contribuições do editor associado e dos revisores para o aprimoramento da versão atual deste artigo.

\section{REFERÊNCIAS}

Abido, M. A. (2003). A novel multiobjective evolutionary algorithm for environmental/economic power dispatch, Electric Power Systems Research, Vol. 65, No. 1, pp. 71-81.

Alander, J. T. (1995). An indexed bibliography of genetic algorithms in power engineering, in Practical Handbook of Genetic Algorithms: New Frontiers, L. Chambers 
Tabela 8: Resultados de convergência para o caso teste 3 (40 unidades geradoras com ponto de válvula) e $P_{D}=10500$ MW.

\begin{tabular}{|c|c|c|c|c|c|}
\hline técnica & $\begin{array}{c}\text { tempo } \\
\text { médio }(\mathrm{s})\end{array}$ & $\begin{array}{c}\text { custo } \\
\text { mínimo }(\$ / \mathrm{h})\end{array}$ & $\begin{array}{c}\text { custo médio } \\
(\$ / \mathrm{h})\end{array}$ & $\begin{array}{c}\text { desvio padrão } \\
\text { do custo }(\$ / \mathrm{h})\end{array}$ & $\begin{array}{c}\text { custo } \\
\text { máximo }(\$ / \mathrm{h})\end{array}$ \\
\hline $\mathrm{SQP}$ & $\mathbf{1 0 , 8 0}$ & 122904,4243 & 124883,7692 & 985,5370 & 126585,2290 \\
\hline $\mathrm{ED}(1)$ & 22,67 & 121813,4385 & 122503,1532 & 501,6266 & 123705,1952 \\
\hline $\mathrm{ED}(2)$ & 31,76 & 121948,7907 & 122843,2914 & 551,1862 & 124274,3533 \\
\hline $\mathrm{ED}(3)$ & 35,09 & 122986,1912 & 124368,9650 & 752,6561 & 126320,1142 \\
\hline $\mathrm{ED}(4)$ & 30,27 & 125016,4174 & 126319,4762 & 1058,4734 & 131158,5790 \\
\hline $\mathrm{ED}(5)$ & 22,85 & 129787,8713 & 123829,6008 & 373,2203 & 124699,9984 \\
\hline $\mathrm{ED}(6)$ & 38,91 & 123137,8314 & 124201,9811 & 401,4912 & 125052,2981 \\
\hline $\mathrm{ED}-\mathrm{SQP}(1) \#$ & 42,05 & 121716,6980 & 121954,8056 & 200,5176 & 122492,2516 \\
\hline $\mathrm{ED}-\mathrm{SQP}(2)^{\#}$ & 73,85 & 121769,1251 & 122303,8502 & 416,9687 & 123634,2098 \\
\hline $\mathrm{ED}-\mathrm{SQP}(3)^{\#}$ & 59,64 & 121810,3545 & 123249,8536 & 629,0922 & 124120,1828 \\
\hline ED-SQP(4) & 20,56 & $\mathbf{1 2 1 7 1 5 , 4 9 0 0}$ & 121918,1695 & 137,4262 & 122267,0088 \\
\hline ED-SQP(5) & 36,86 & 121723,4893 & $\mathbf{1 2 1 8 9 0 , 1 6 3 0}$ & $\mathbf{1 2 0 , 1 2 9 4}$ & $\mathbf{1 2 2 1 8 9 , 3 2 6 2}$ \\
\hline ED-SQP(6) & 38,90 & 121770,7606 & 122859,9637 & 588,7051 & 124449,2637 \\
\hline
\end{tabular}

\# foi usada na ED uma população de 30 indivíduos e $G_{\max }=1000$.

Tabela 9: Melhor solução para o estudo de caso 3 obtido usando ED-SQP(1-6) e SQP.

\begin{tabular}{|c|c|c|c|c|c|c|c|}
\hline potência & geração & potência & geração & potência & geração & potência & geração \\
\hline$P_{1}$ & 110,8005 & $P_{11}$ & 168,7978 & $P_{21}$ & 523,2797 & $P_{31}$ & 190,0000 \\
\hline$P_{2}$ & 110,8007 & $P_{12}$ & 168,7997 & $P_{22}$ & 523,2788 & $P_{32}$ & 190,0000 \\
\hline$P_{3}$ & 97,3993 & $P_{13}$ & 125,0005 & $P_{23}$ & 523,2793 & $P_{33}$ & 190,0000 \\
\hline$P_{4}$ & 179,7329 & $P_{14}$ & 394,2792 & $P_{24}$ & 523,2797 & $P_{34}$ & 200,0000 \\
\hline$P_{5}$ & 87,8001 & $P_{15}$ & 394,2796 & $P_{25}$ & 523,2798 & $P_{35}$ & 164,8009 \\
\hline$P_{6}$ & 140,0000 & $P_{16}$ & 304,5190 & $P_{26}$ & 523,2796 & $P_{36}$ & 200,0000 \\
\hline$P_{7}$ & 259,6012 & $P_{17}$ & 489,2794 & $P_{27}$ & 10,0004 & $P_{37}$ & 110,0000 \\
\hline$P_{8}$ & 300,0000 & $P_{18}$ & 489,2800 & $P_{28}$ & 10,0001 & $P_{38}$ & 110,0000 \\
\hline$P_{9}$ & 284,6004 & $P_{19}$ & 511,2789 & $P_{29}$ & 10,0002 & $P_{39}$ & 110,0000 \\
\hline$P_{10}$ & 130,0002 & $P_{20}$ & 511,2796 & $P_{30}$ & 96,7133 & $P_{40}$ & 511,2794 \\
\hline & & & & & & $\sum_{i=1} P_{i}$ & 10500,0000 \\
\hline
\end{tabular}

(ed.), Boca Raton, CRC Press, Vol. 2.

Arsham, H.; Gradisar, M.; Stemberger, M. I. (2003). Linearly constrained global optimization: a general solution algorithm with applications, Applied Mathematics and Computation, Vol. 134, No. 2-3, pp. 345-361.

Attaviriyanupap, P.; Kita, H.; Tanaka, E.; Hasegawa, J. (2004). A fuzzy-optimization approach to dynamic economic dispatch considering uncertainties, IEEE Transactions on Power Systems, Vol. 19, No. 3, pp. 12991307.
Bäck, T.; Fogel, D. B.; Michalewicz, Z. (eds.) (1997). Handbook of evolutionary computation, Bristol, Philadelphia: Institute of Physics Publishing, NY, Oxford: Oxford University Press.

Boggs, P. T.; Tolle, J. W. (2000). Sequential quadratic programming for large-scale nonlinear optimization, Journal of Computational and Applied Mathematics, Vol. 124, No. 1-2, pp. 123-137.

Chang, C. S.; Fu, W. (1998). Stochastic multi-objective generation dispatch of combined heat and power systems, IEE Proc. Control, Generation, Transmission and Distribution, Vol. 145, No. 5, pp. 585-591. 
Tabela 10: Análise comparativa com os resultados apresentados na literatura para a função custo $f$ e os obtidos neste trabalho.

\begin{tabular}{|c|c|c|c|}
\hline técnica & caso 1 & caso 2 & caso 3 \\
\hline algoritmo genético (Walters \& Sheble, 1993) & 8237,60 & - & - \\
\hline programação evolucionária (Sinha et al., 2003) & $\mathbf{8 2 3 4 , 0 7}$ & 17994,07 & 122624,35 \\
\hline nuvem de partículas (Victoire \& Jeyakumar, 2004) & $\mathbf{8 2 3 4 , 0 7}$ & 18030,72 & 122930,45 \\
\hline nuvem de partículas modificado (Park et al., 2005) & $\mathbf{8 2 3 4 , 0 7}$ & - & 122252,265 \\
\hline $\begin{array}{c}\text { programação evolucionária híbrida com SQP } \\
\text { (Victoire \& Jeyakumar, 2004) }\end{array}$ & $\mathbf{8 2 3 4 , 0 7}$ & 17991,03 & 122379,63 \\
\hline $\begin{array}{c}\text { nuvem de partículas híbrida com SQP } \\
\text { (Victoire \& Jeyakumar, 2004) }\end{array}$ & $\mathbf{8 2 3 4 , 0 7}$ & 17969,93 & 122094,67 \\
\hline $\begin{array}{c}\text { melhor resultado deste artigo } \rightarrow \\
\text { usando a(s) técnica(s) } \rightarrow\end{array}$ & $\begin{array}{c}\mathbf{8 2 3 4 , 0 7 4 0} \\
\text { SQP e ED-SQP(1-6) }\end{array}$ & $\begin{array}{c}\mathbf{1 7 9 6 4 , 0 7 5 8} \\
\text { SQP e ED-SQP(1-6) }\end{array}$ & $\begin{array}{c}\mathbf{1 2 1 7 1 5 , 4 9 0 0} \\
\text { ED-SQP(4) }\end{array}$ \\
\hline
\end{tabular}

Chen, C. L.; Wang. C. L. (1993). Branch-and-bound scheduling for thermal generating units, IEEE Transactions on Energy Conversion, Vol. 8, No. 2, pp. 184-189.

Chen, P. -H.; Chang, H. -C. (1995). Large-scale economic dispatch by genetic algorithm, IEEE Transactions on Power Systems, Vol. 10, No. 4, pp. 1919-1925.

Cheng, S. -L.; Hwang, C. (2001). Optimal approximation of linear systems by a differential evolution algorithm, IEEE Transactions on Systems, Man, and Cybernetics ? Part A: Systems and Humans, Vol. 31, No. 6, pp. 698-707.

Chowdhury, B. H.; Rahman, S. (1990). A review of recent advances in economic dispatch, IEEE Transactions on Power Apparatus and Systems, Vol. 5, No. 4. pp. 12481259.

Coelho, L. S.; Mariani, V. C. (2006). Particle swarm optimization with quasi-Newton local search for solving economic dispatch problem, Proceedings of IEEE Systems, Man, and Cybernetics Conference, Taipei, Taiwan.

Das, D. B.; Patvardhan, C. (1999). Solution of economic load dispatch using real coded hybrid stochastic search, International Journal of Electric Power and Energy Systems, Vol. 21, No. 3, pp. 165-170.

El-Keib, A. A.; Ding, H. (1994). A fast environmentally constrained economic dispatch using linear programming, Electric Power Systems Research, Vol. 29, No. 3, pp. 155-159.

Farag, A.; Al-Baiyat, S.; Cheng, T. C. (1995). Economic load dispatch multiobjective optimization procedures using linear programming techniques, IEEE Transactions on Power Systems, Vol. 10, No. 2, pp. 731-738.
Fletcher, R. (1987). Practical methods of optimization, 2nd edition, John Wiley \& Sons, New York, NY.

Granville, S. (1992). Optimal reactive dispatch through interior point methods, IEEE Summer Meeting, Paper No. 92 SM 416-8 PWRS, Seattle, WA.

IEEE Committee Report (1971). Present practice in the economic operation of power systems, IEEE Transactions on Power Apparatus and Systems, Vol. 90, pp. 17681775.

Jabayarathi, T.; Sadasivam, G.; Ramachandran, V. (2000). Evolutionary programming-based multi area economic dispatch with tie line constraints, Electric Machines and Power Systems, Vol. 28, No. 12, pp. 1165-1176.

Jin, X.; Reynolds, R. G. (1999). Using knowledge-based evolutionary computation to solve nonlinear constraint optimization problems: a cultural algorithm approach, IEEE Congress on Evolutionary Computation, Washington, DC, pp. 1672-1678.

Ku, K. W. C.; Mak, M. W.; Siu, W. -C. (2000). A study of the Lamarckian evolution of recurrent neural networks, IEEE Transactions on Evolutionary Computation, Vol. 4, No. 1, pp. 31-41.

Kumarappan, N.; Mohan, M. R. (2003). Fuel restricted short term economic dispatch using evolutionary programming for utility system, International Journal of Electrical Power and Energy Systems, Vol. 25, No. 10, pp. 821-827.

Lee, K. Y.; Park, Y. M.; Ortiz, J. L. (1984). Fuel cost minimization for both real and reactive power dispatches, IEE Proc. Control, Generation, Transmission and Distribution, Vol. 131, No. 3, pp. 85-93. 
Lee, K. Y.; Yome, A. S-.; Park, J. H. (1998). Adaptive Hopfield neural networks for economic load dispatch, IEEE Transactions on Power Systems, Vol. 13, No. 2, pp. 519-526.

Lee, S. C.; Kim, Y. H. (2002). An enhanced Lagrangian neural network for the ELD problems with piecewise quadratic cost functions and nonlinear constraints, Electric Power Systems Research, Vol. 60, No. 3, pp. 167-177.

Li, F.; Morgan, R.; Williams, D. (1997). Hybrid genetic approaches to ramping rate constrained dynamic economic dispatch, Electric Power Systems Research, Vol. 43, No. 2, pp. 97-103.

Liang, Z. X.; Glover, J. D. (1992). A zoom feature for a programming solution to economic dispatch including transmission losses, IEEE Transactions on Power Systems, Vol. 7, No. 3, pp. 544-550.

Lin, C. E.; Viviani, G. L. (1984). Hierarchical economic dispatch for piecewise quadratic cost functions, IEEE Transactions on Power Apparatus and Systems, Vol. 103, No. 6, pp. 1170-1175.

Lin, W. M.; Cheng, F. S.; Tsay, M. T. (2002). An improved tabu search for economic dispatch with multiple minima, IEEE Transactions on Power Systems, Vol. 17, No. 1, pp. 108-112.

Miranda, V.; Srinivasan, D.; Proença, L. M. (1998). Evolutionary computation in power systems, Electric Power Energy Systems, Vol. 20, No. 2, pp. 89-98.

Moscato, P.; Norman, M. G. (1992). A 'memetic' approach for the travelling salesman problem ? implementation of a computational ecology for combinatorial optimisation on message-passing systems, Proceedings of the International Conference on Parallel Computing and Transputer Applications, IOS Press, Amsterdam, pp. 177-186.

Orero, S. O.; Irving, M. R. (1996). Economic dispatch of generators with prohibited operating zones: a genetic algorithm approach, IEE Proc. Control, Generation, Transmission and Distribution, Vol. 143, No. 6, pp. 529-534.

Park, J. -B.; Lee, K. -S.; Shin, J. -R.; Lee,, K. Y. (2005). A particle swarm optimization for economic dispatch with nonsmooth cost function, IEEE Transactions on Power Systems, Vol. 20, No. 1, pp. 34-42.

Park, J. H.; Kim, Y. S.; Eom, I. K.; Lee, K. Y. (1993). Economic load dispatch for piecewise quadratic cost function using Hopfield neural network, IEEE Transactions on Power Systems, Vol. 8, No. 3, pp. 1030-1038.
Park, Y.; Won, J.; Park, J.; Kim, D. (1999). Generation expansion planning based on an advanced evolutionary programming, IEEE Transactions on Power Systems, Vol. 14, No. 1, pp. 299-305.

Reynolds, R. G. (1994). An introduction to cultural algorithms, Proceedings of the 3rd Annual Conference on Evolutionary Programming, Sebald, A. V.; Fogel, L. J. (eds), World Scientific, River Edge, NJ, pp. 131-139.

Rodríguez-Toral, M. A.; Morton, W.; Mitchell, D. R. (2001). The use of new SQP methods for the optimization of utility systems, Computers and Chemical Engineering, Vol. 25, No. 2-3, pp. 287-300.

Ruangpayoongsak, N.; Ongsakul, W.; Runggeratigul, S. (2002). Constrained economic dispatch by combined genetic and simulated annealing algorithm, Electric Power Components and Systems, Vol. 30, No. 9, pp. 917-931.

Sheble, G. B.; Brittig, K. (1995). Refined genetic algorithmeconomic dispatch example, IEEE Transactions on Power Systems, Vol. 10, No. 1, pp. 117-124.

Shoults, R. R.; Venkatesh, S. V. (1986). A dynamic programming based method for developing dispatch curves when incremental heat rate curves are nonmonotonically increasing, IEEE Transactions on Power Systems, Vol. 1, No. 1, pp. 10-16.

Sinha, N.; Chakrabarti, R.; Chattopadhyay, P. K. (2003). Evolutionary programming techniques for economic load dispatch, IEEE Transactions on Evolutionary Computation, Vol. 7, No. 1, pp. 83-94.

Song, Y. H.; Wang, G. S.; Wang, P. Y.; Johns, A. T. (1997). Environmental/economic dispatch using fuzzy logic controlled genetic algorithms, IEE Proc. Control, Generation, Transmission and Distribution, Vol. 144, No. 4, pp. 377-382.

Storn, R. (1997). Differential evolution ? a simple and efficient heuristic for global optimization over continuous spaces, Journal of Global Optimization, Vol. 11, No. 4, pp. 341-359.

Storn, R. (1999). System design by constraint adaptation and differential evolution, IEEE Transactions on Evolutionary Computation, Vol. 3, No. 1, pp. 22-33.

Storn, R.; Price, K. (1995). Differential evolution: a simple and efficient adaptive scheme for global optimization over continuos spaces, Technical Report TR-95-012, International Computer Science Institute, Berkeley. 
Su, C. -T.; Chiang, C. -L. (2004). An incorporated algorithm for combined heat and power economic dispatch, Electric Power Systems Research, Vol. 69, No. 2-3, pp. 187 195.

Vasconcelos, J. A.; Saldanha, R. R.; Krähenbühl, L.; Nicolas, A. (1999). Genetic algorithm coupled with a deterministic method for optimization in electromagnetics, IEEE Transactions on Magnetics, Vol. 33, No. 3, pp. 1860-1863.

Venkatesh, P.; Gnanadass, R.; Prasad, P. N. (2003). Comparison and application of evolutionary programming techniques to combined economic emission dispatch with line flow constraints, IEEE Transactions on Power Systems, Vol. 10, No. 4, pp. 688-697.

Victoire, T. A. A.; Jeyakumar, A. E. (2004). Hybrid PSOSQP for economic dispatch with valve-point effect, Electric Power Systems Research, Vol. 71, No. 1, pp. 51-59.

Walters, D. C.; Sheble, G. B. (1993). Genetic algorithm solution of economic dispatch with valve point loading, IEEE Transactions on Power Systems, Vol. 8, No. 3, pp. 1325-1332.

Wang, C.; Shahidepour, S. M. (1992). A decomposition approach to non-linear multi-area generation scheduling with tie line constraints using expert systems, IEEE Transactions on Power Systems, Vol. 7, No. 4, pp. 1409-1418.

Wellock, C.; Ross B. J. (2001). An examination of Lamarckian genetic algorithms, Genetic and Evolutionary Computation Conference Late Breaking Papers, San Francisco, CA, USA, pp. 474-481.

Whitley, D. (2001). An overview of evolutionary algorithms: practical issues and common pitfalls, Information and Software Technology, Vol. 43, No. 14, pp. 817-831.

Whitley, D.; Gordon, V. S.; Mathias, K. (1994). Lamarckian evolution, the Baldwin effect and function optimization, Parallel Problem Solving from Nature, Davidor, Y.; Schwefel, H. -P.; Manner, R. (eds.), SpringerVerlag, pp. 6-15.

Wong, K. P.; Fung, C. C. (1993). Simulated-annealing based economic dispatch algorithm, IEE Proc. Pt. C, Vol. 140, No. 6, pp. 509-515.

Wong, K. P.; Wong, Y. W. (1994). Genetic and genetic/simulated-annealing approaches to economic dispatch, IEE Proc. Control, Generation, Transmission and Distribution, Vol. 141, No. 5, pp. 507-513.
Wong, K. P.; Yuryevich, J. (1998). Evolutionary programming-based algorithm for environmentally constrained economic dispatch, IEEE Transactions on Power Systems, Vol. 13, No. 2, pp. 301-306.

Wood, A. J.; Wollenberg, B. F. (1994). Power generation, operation and control, New York, John Wiley \& Sons.

Xu, J. X.; Chang, C. S.; Wang, X. W. (1996). Constrained multiobjective global optimization of longitudinal interconnected power system by genetic algorithm, IEE Proc. Control, Generation, Transmission and Distribution, Vol. 143, No. 5, pp. 435-446.

Yalcinoz, T.; Short, M. J. (1998). Neural networks approach for solving economic dispatch problems with transmission capacity constraints, IEEE Transactions on Power Systems, Vol. 13, No. 2, pp. 307-313.

Yang, H. -T.; Yang, P. -C.; Huang, C. -L. (1996). Evolutionary programming based on economic dispatch for units with non-smooth incremental fuel cost functions, IEEE Transactions on Power Systems, Vol. 11, No. 1, pp. 112-118.

Yang. H. T.; Chen, S. L. (1989). Incorporating a multicriteria decision procedure into the combined dynamic programming/production simulation algorithm for generation expansion planning, IEEE Transactions on Power Systems, Vol. 4, No. 1, pp. 165-175. 